\title{
Long-term effect on optic nerve of silicone oil tamponade in rabbits: histological and EDXA findings
}

\author{
A Saitoh ${ }^{1}, \mathrm{H}$ Taniguchi ${ }^{1}, \mathrm{H}$ Gong ${ }^{1}$, A Ohira ${ }^{1}$, \\ T Amemiya ${ }^{1}$ and $\mathrm{T}$ Baba $^{2}$
}

\begin{abstract}
Purpose Side-effects after intravitreal use of silicone oil (SO) are not well defined and elucidated. The object of this study was to examine the influence and toxicity of $\mathrm{SO}$ on the optic nerve after vitrectomy with SO tamponade.
\end{abstract}

Methods We injected medical grade $\mathrm{SO}$ and emulsified SO into rabbit eyes after gasmediated vitreous compression and examined the eyes by light microscopy (LM), transmission electron microscopy (TEM) and energy dispersive X-ray analysis (EDXA) (point analysis and area analysis) 6 months after injection. We compared the findings in the non-treated eyes and eyes with only gasmediated vitreous compression with those in SO-injected eyes.

Results Vacuole-like structures were seen in the optic nerve posterior to the lamina cribrosa. In the group treated with only gasmediated vitreous compression, the myelin structures were shown by TEM to be destroyed and replaced by glial tissue, while in groups injected with medical grade or emulsified SO severe destruction of the myelin sheath (myelinolysis) was observed. Silicone was identified at the electron-dense edges of the vacuoles by EDXA point analysis, but not in the vacuoles without electron-dense deposits. Dots of Si K alpha were not seen in the control groups, and dense dots were observed in SO-injected groups, by EDXA area analysis.

Conclusions Some of the vacuoles might be artefacts caused by insufficient fixation or the operative procedure, but TEM showed almost no artefacts in the control optic nerve. Thus, most vacuoles may be SO storage sites. SO uptake into the optic nerve might play a role in the pathogenesis of optic nerve atrophy after SO injection.
Eye (2002) 16, 171-176. DOI: 10.1038/

sj/EYE/6700099

Keywords: EDXA; emulsified silicone oil; gasmediated vitreous compression; mapping; myelin; silicone oil; vacuole

\section{Introduction}

Silicone oil (SO: polydimethylsiloxane) tamponade has been used in complicated cases such as proliferative vitreoretinopathy, giant retinal tears and retinal detachment caused by diabetic retinopathy. ${ }^{1-7}$ We sometimes observe optic nerve atrophy in eyes treated with SO tamponade. Many papers have reported the influence and toxicity of SO on retinal tissues, but to our knowledge there are only a few histological descriptions of the influence and toxicity of SO on the optic nerve. ${ }^{8-11}$ Clinically it is thought that the period of SO tamponade should be limited to 6 months. To study the influence and toxicity of long-term SO tamponade on the optic nerves, we injected medical grade $\mathrm{SO}$ and emulsified SO into rabbit eyes after gasmediated vitreous compression, and 6 months later examined them by light microscopy (LM), transmission electron microscopy (TEM) and energy dispersive X-ray analysis (EDXA) (point analysis and area analysis) with TEM and scanning electron microscopy (SEM). SOtreated eyes were compared with untreated eyes and eyes with only gas-mediated vitreous compression.

Materials and methods

\section{Materials}

Twenty-five albino rabbits (JRW line), weighing $2.5-3.5 \mathrm{~kg}$ at the beginning of the
'Department of Ophthalmology and Visual Sciences

Nagasaki University School of Medicine

Nagasaki, Japan

${ }^{2}$ Technology Center of Nagasaki

Nagasaki, Japan

Correspondence:

A Saitoh MD

Department of

Ophthalmology and Visual Sciences

Nagasaki University School of Medicine

1-7-1 Sakamoto

Nagasaki City

852-8501, Japan

Tel: +81958497345

Fax: +81958497347

E-mail: tamemiya@

net.nagasaki-u.ac.jp

Sponsorship/commercial or proprietary interest/ financial interest: None

Received: 5 September 2000

Accepted in revised form: 18 May 2001 
experiment, were maintained at the Laboratory Animal Centre for Biomedical Research, Nagasaki University School of Medicine and were treated in accordance with the ARVO Statement on the Use of Animals in Ophthalmic and Vision Research. Eyes from 25 rabbits were tested: 10 in group C, a control group with no surgical procedure; five in group G, treated with only gas-mediated vitreous compression; five in group M6, examined 6 months after the injection of medical grade SO; and five in group E6, examined 6 months after the injection of emulsified SO. Medical grade SO was 1000 Cs SO (Richard-James, Peabody, MA, USA). For the preparation of emulsified SO, $3.0 \mathrm{~g}$ of $100 \mathrm{cS}$ SO (Toshiba Silicone, Tokyo, Japan), $0.2 \mathrm{~g}$ of egg lecithin (Wako-junyaku, Osaka, Japan) and $10 \mathrm{ml}$ of distilled water were mixed, and high-intensity ultrasound sonication was performed for $30 \mathrm{~min}$.

\section{Surgical methods}

Before all operative procedures the rabbits were anaesthetised with an auricular venous injection of 20 $30 \mathrm{mg} / \mathrm{kg}$ pentobarbital. Topical anaesthesia with procaine hydrochloride eyedrops was next applied, and the pupils were dilated with eyedrops of $0.5 \%$ tropicamide and phenylephrine hydrochloride. The eyes were examined pre-operatively by indirect ophthalmoscopy to confirm a normal appearance.

Space for the SO injection was made by the vitreous compression technique with perfluoropropane gas $\left(\mathrm{C}_{3} \mathrm{~F}_{8}\right) .{ }^{12}$ In group $\mathrm{G}$, the gas was exchanged for $1.5 \mathrm{ml}$ of balanced salt solution after 3 days. In groups M6 and E6, the gas was exchanged for $1.5 \mathrm{ml}$ of SO after 3 days. The contralateral eyes were spared. After all operative procedures ofloxacin eye drops were applied topically.

\section{Histological and EDXA examination}

All rabbits were killed 6 months after injection. Under the above-mentioned anaesthesia, the eyes and optic nerves were enucleated. In groups M6 and E6, the optic nerves were cut at the insertion site of the sclera into the eyeball to avoid SO dispersion, and fixed in $10 \%$ neutral formalin. In groups $C$ and $G$, optic nerves were cut with sparing of the optic nerve head and fixed in $10 \%$ neutral formalin. Specimens for LM and EDXA with SEM were dehydrated in a series of ethanols and embedded in paraffin. Some of the serial cross-sections were coated with carbon and examined with a JSM-6400F scanning electron microscope (JEOL, Tokyo, Japan) with EDXA9800 X-ray analyser (Philips) under the same conditions $(\times 270,20 \mathrm{keV}$, uptake time: 200 s). Point analysis and X-ray distribution images (area analysis) with SEM were performed. Specimens for TEM for cytological observation were fixed in $4 \%$ glutaraldehyde in $0.05 \mathrm{M}$ cacodylate buffer for $1 \mathrm{~h}$ and postfixed in $1 \%$ osmium tetroxide in veronal acetate buffer for $1 \mathrm{~h}$ after an overnight wash with $0.44 \mathrm{M}$ sucrose. Specimens for EDXA with TEM were fixed in $4 \%$ glutaraldehyde in $0.05 \mathrm{M}$ cacodylate buffer for $1 \mathrm{~h}$ but not postfixed in $1 \%$ osmium tetroxide in veronal acetate buffer. The fixed materials were dehydrated through a series of ethanols and embedded in Luveak 812 (Nakalai Tesque, Kyoto, Japan). Ultrathin sections were cut with a Porter-Blum MT2 microtome. Unstained sections for EDXA with TEM were examined with a JEM-1210 (JEOL, Tokyo, Japan) with DX-4 X-ray dispersion analytical equipment (Philips) under the same conditions (20 keV, uptake time: $200 \mathrm{~s}$ ). The specimens for observation were stained with uranyl acetate and lead citrate and observed with a JEM-1210 transmission electron microscope (JEOL, Tokyo, Japan). Because of limited equipment, area analysis could not be done with SEM.

\section{Results}

\section{LM findings}

In group $C$, vacuoles were not seen (Figure 1). But in groups G, M6 and E6, vacuole-like structures were seen in the optic nerves posterior to the lamina cribrosa (Figure 2).

\section{TEM findings}

In group $C$ regular myelin structures were observed and the nerve bundles were surrounded by a continuous layer of subseptal astrocytes (Figure 3a). In

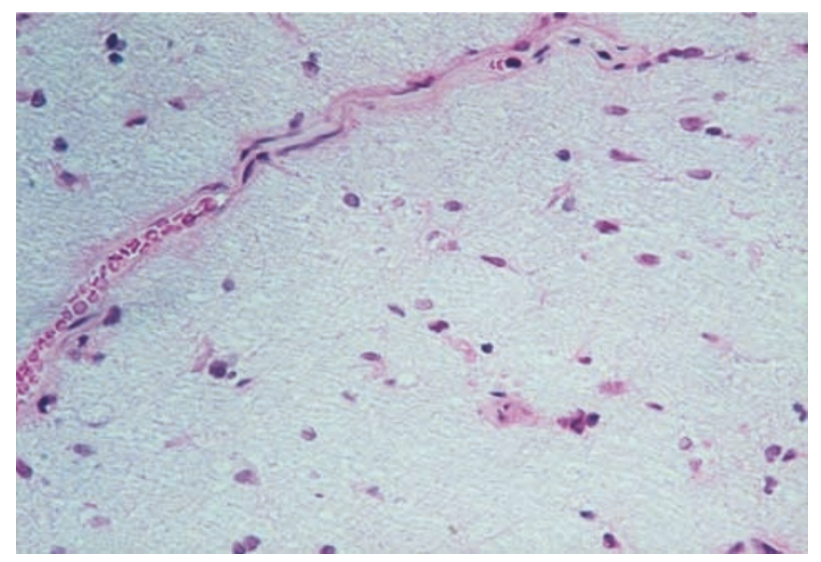

Figure 1 Light micrograph of the optic nerve of a control rabbit (haematoxylin-eosin stain, $\times 400$ ). Vacuole-like structures are not seen. 


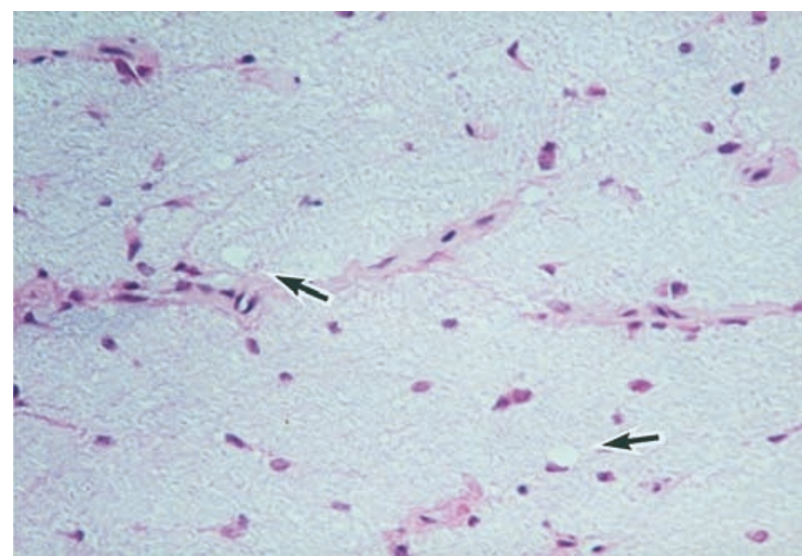

Figure 2 Light micrograph of the optic nerve head of a control group G. Vacuole-like structures (arrows) are seen in the optic nerve head posterior to the lamina cribrosa (haematoxylin-eosin stain, $\times 400)$.

a

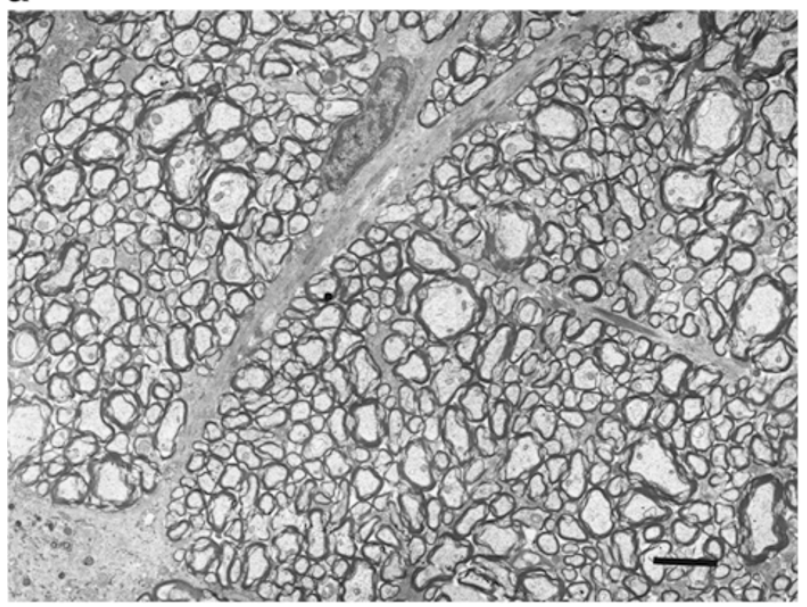

C

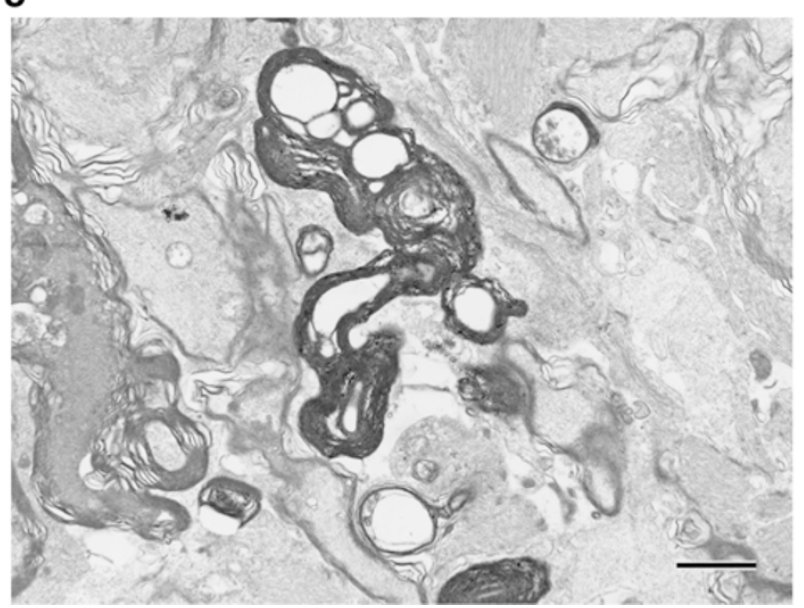

group $\mathrm{G}$ the myelin structures were destroyed and replaced by glial tissue (Figure 3b), while in groups M6 and E6, severe destruction of the myelin sheath (myelinolysis) was observed (Figure 3c, d). There was no significant difference in TEM findings between groups M6 and E6. In axons of the myelinated nerve fibres, mitochondria and neurotubules were destroyed. Vacuoles with dense contour were scattered in the astrocyte cytoplasm.

\section{EDXA (point analysis) with TEM findings}

In group C, EDXA (point analysis) with TEM showed no $\mathrm{K}$ alpha peak of $\mathrm{Si}$ in any intracytoplasmic

organelles including vesicular structures. In group M6, EDXA with TEM was performed on LUVEAK 812 only (control), and on axons, myelin sheaths, vacuoles and electron-dense deposits in the vacuoles (Figures 4, 5).

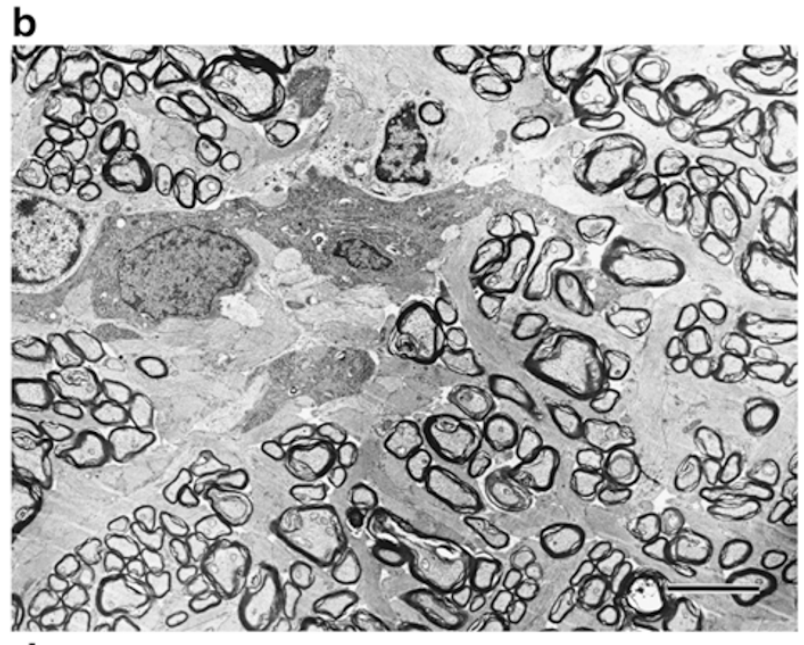

\section{d}

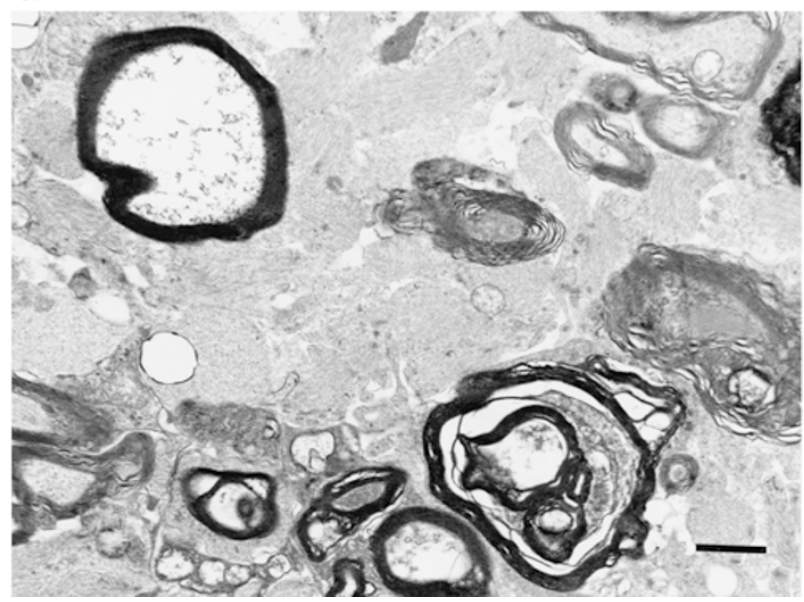

Figure 3 Transmission electron microscopic findings of an optic nerve. The myelin sheath in the control group is normal (a). Group G shows a prominent decrease of myelinated nerve fibres and destruction of the myelin sheath posterior to the lamina cribrosa (b). Group M6 (c) and group E6 (d) show severe destruction of the myelin sheath. The scale bars represent $2 \mu \mathrm{m}$ (a), $4 \mu \mathrm{m}$ (b) or $1 \mu \mathrm{m}$ $(\mathrm{c}, \mathrm{d})$. 


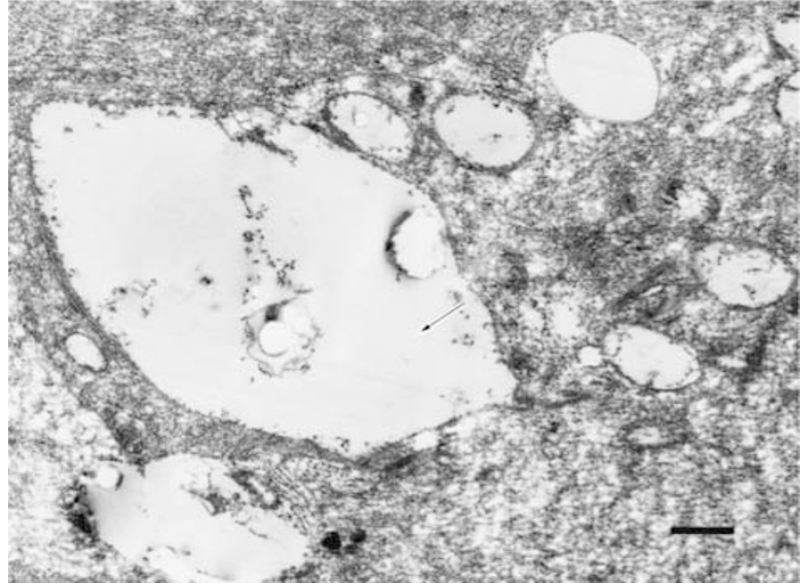

Figure 4 Transmission electron microscopic appearance of a vacuole without electron-dense deposits. The scale bar represents $500 \mathrm{~nm}$ and the arrow shows the analysis point.

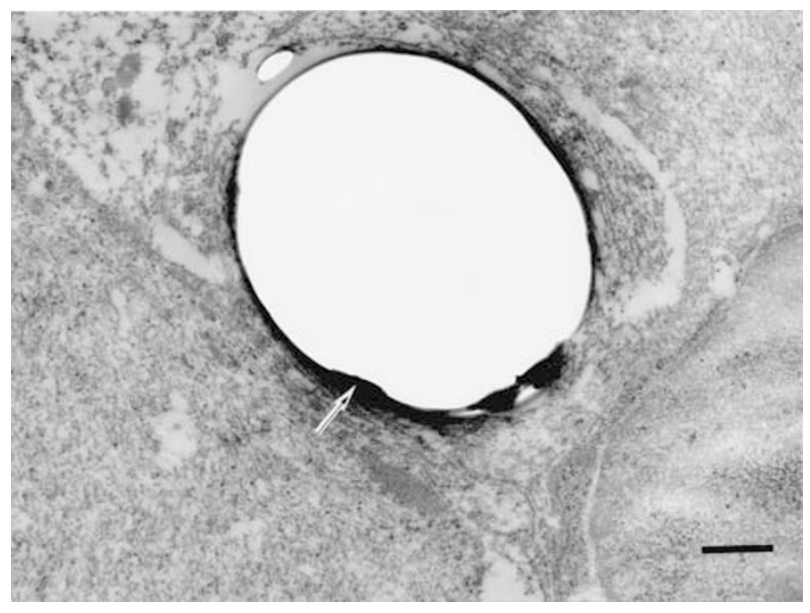

Figure 5 Transmission electron microscopic appearance of a vacuole with electron-dense deposits. The edge of the vacuole is curled. The scale bar represents $500 \mathrm{~nm}$ and the arrow shows the analysis point.

Only in the electron-dense deposits of the vacuoles was $\mathrm{K}$ alpha of $\mathrm{Si}$ observed (Figure 6). In the axons, myelin sheaths, centres of vacuoles, and edges of vacuoles without electron-dense deposits, no $\mathrm{K}$ alpha peaks of Si were observed.

\section{EDXA (point analysis and area analysis) with SEM findings}

We examined the $\mathrm{X}$-ray distribution images (area analysis) of Si K alpha on the surface of the sectioned optic nerves as well as point analysis with EDXA with SEM. In group $\mathrm{C}$, neither $\mathrm{K}$ peaks of $\mathrm{Si}$ nor dots were seen (Figure 7a). That is, there is too little $\mathrm{Si}$ to be detected in the normal optic nerve. In groups M6 and E6, dense dots of $\mathrm{K}$ alpha of $\mathrm{Si}$ were seen immediately

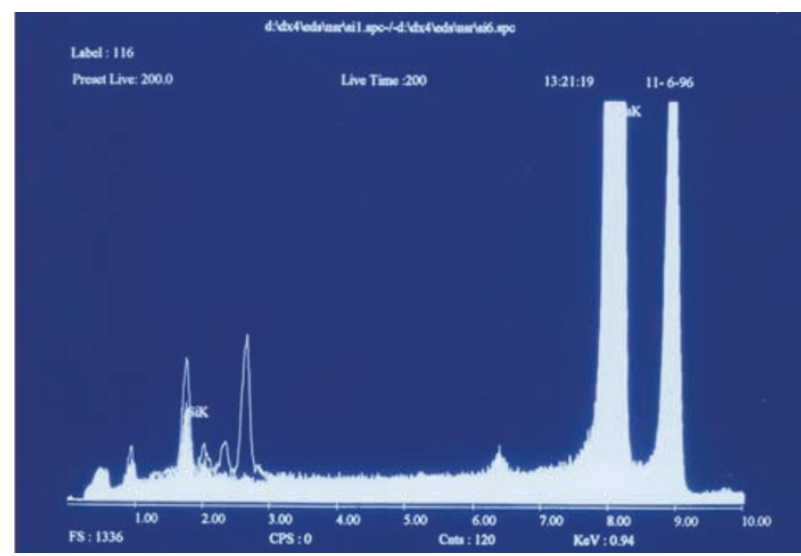

Figure 6 Electron-dense deposits at the edge of a vacuole show the $\mathrm{K}$ alpha peak of $\mathrm{Si}$ (line). The white region shows the peak curve of the analysis of LUVEAK 812 (control)

posterior to but not $1 \mathrm{~mm}$ beyond the lamina cribrosa (Figure $7 b, c)$. Si injection into the vitreous cavitry was absorbed into the optic nerve. There was no significant difference in the dot density between M6 and E6.

\section{Discussion}

Histological studies of enucleated human eyes following long-term SO tamponade and morphological and histochemical analysis of experimental SO tamponade have shown side-effects including intracytoplasmic vacuole formation, intraocular foreign body reaction, and degenerative changes in ganglion and photoreceptor cells and the plexiform layer. ${ }^{13-19}$ Some investigators have regarded the vacuoles as artefacts caused by insufficient fixation or the operative procedure, ${ }^{18-20}$ but others have considered them to be deposits of SO. Ohira et al ${ }^{21,22}$ injected SO with phthalocyanine blue dye into rabbit eyes and observed vacuoles surrounded by phthalocyanine blue dye deposits; they suggested that the vacuoles represent SO droplets.

Eckardt et al ${ }^{14}$ examined the histological characteristics of 11 SO-injected eyes (cornea, four cases; iris, three cases; retina, eight cases; retinal pigment epithelium and optic nerves, one case) 6 months to 3.5 years after SO injection. They observed vacuoles in the iris, cornea and retina. Using EDXA (point analysis), they could identify vacuoles in the retina as storage sites of SO more than 6 months after $\mathrm{SO}$ injection, but found no silicone in the one optic nerve they examined. Knorr et $a l^{9}$ studied 36 enucleated eyes with SO tamponade clinically and histologically, and observed glaucomatous atrophy of the optic nerve (56\%). They found vacuoles in the optic nerve in eight of nine eyes and concluded that there 
a

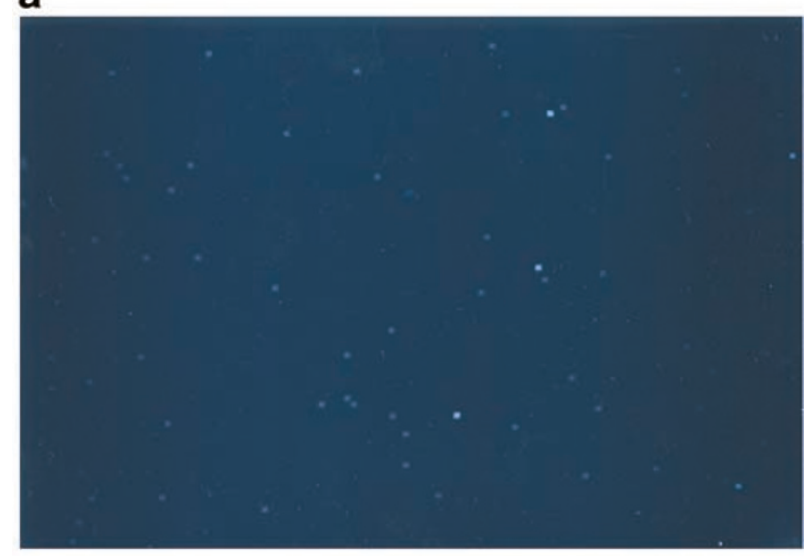

b

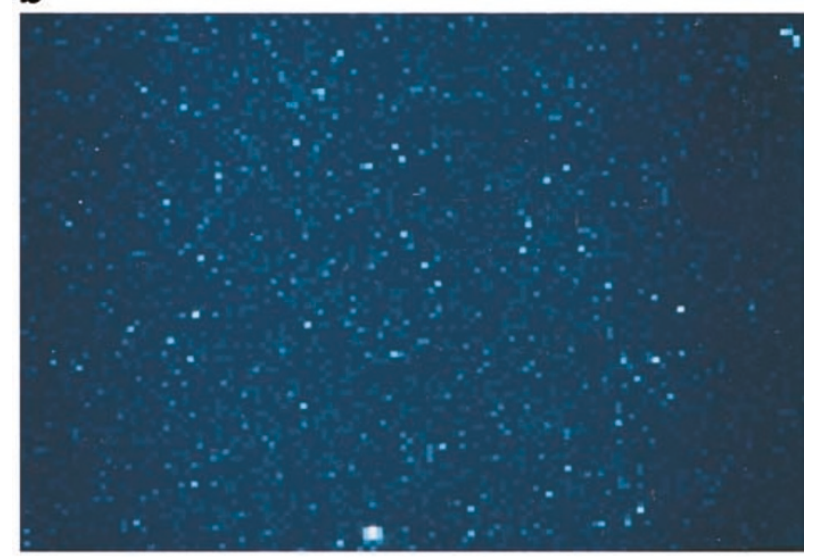

c

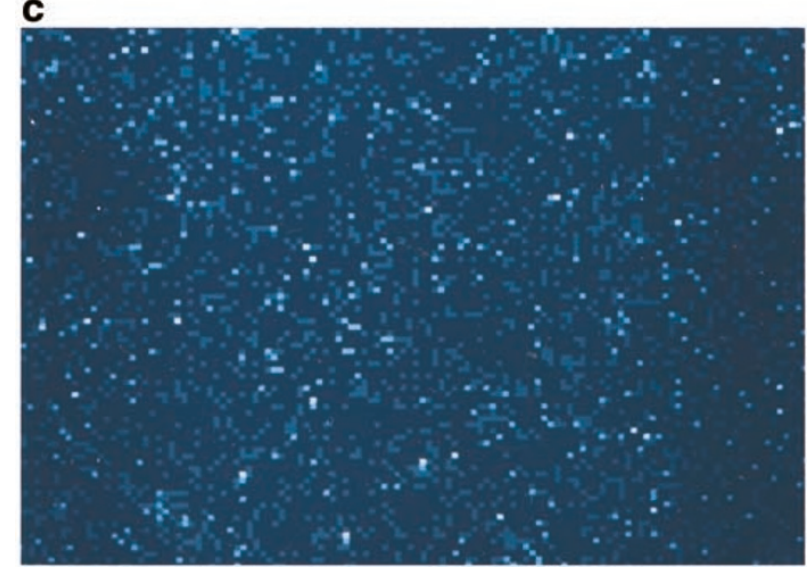

Figure 7 In group C, no distinct dots are seen (a). In groups M6 (b) and E6 (c), dense dots of K alpha of Si are seen.

was no correlation between the duration and viscosity of intraocular $\mathrm{SO}$ and the presence of vacuoles in the optic nerve. It was speculated that some of the vacuoles were artefacts caused by insufficient fixation or the operative procedure. However, EM showed no abnormalities in the control optic nerve. Thus, most vacuoles may be SO storage sites.
K peaks of Si were observed only in the electrondense deposits in the vacuoles and not in the centres or edges of vacuoles without electron-dense deposits. ${ }^{23}$ $\mathrm{SO}$ particles in ocular tissues have been believed to escape easily during fixation and hydration. Stained with osmium or uranyl acetate and lead citrate, SO appears as electron-dense deposits. ${ }^{22}$ Our results suggest that, while vacuoles without electron-dense deposits represent degeneration, oedema or artefact, electron-dense deposits at the edge of the vacuoles may represent residual silicone remaining after the preparation of tissues for histological observation.

Whether $\mathrm{SO}$ is toxic to the retina remains controversial. Several reports on this issue have been published, but to our knowledge there have been none with the main purpose of determining whether $\mathrm{SO}$ is toxic to the optic nerve. Wenkel and Nauman ${ }^{10}$ observed bubble-like changes along the optic nerve in an enucleated eye filled with SO. Eller $e t$ al ${ }^{11}$ reported that a patient who underwent vitrectomy with SO tamponade for the treatment of retinal detachment secondary to cytomegalovirus retinitis was found to have a foreign substance in his lateral ventricles and along the intracranial portion of his optic nerve, and concluded that such a substance might be $\mathrm{SO}$ according to computed tomography and magnetic resonance imaging. But they did not confirm their findings with EDXA. Our report is the first to confirm experimentally with EDXA SO migration into the optic nerve posterior to the lamina cribrosa. We used gasmediated vitreous compression in this study.

Demyelination was significantly more severe in the SOinjected groups than in group G (eyes injected only with balanced salt solution after gas-mediated vitreous compression).

The optic nerve damage in our experiment is therefore thought to be due to SO injection. Thus, demyelination and vacuoles in the optic nerve may be evidence of the toxic effect of SO.

We used X-ray distribution images in this study. As the electron beam scans the surface, the X-ray analyser searches for a particular element of interest and, whenever an X-ray of that energy is detected, a bright dot appears at the corresponding point on the display of the SEM, and the density of the dots in each area is related to the concentration of the element. In our study, dots of Si Kalpha were not seen in the controls (groups $\mathrm{C}$ and G), and the dense dots of Si K alpha were observed 6 months after the injection of medical grade or emulsified SO. TEM and X-ray distribution images suggested that $\mathrm{SO}$ uptake by the optic nerve might play a role in the pathogenesis of optic nerve atrophy after SO injection. Possible mechanisms of optic nerve atrophy after SO injection are: (i) Surgical 
manipulation mechanically injures the nerve fibre layer and causes degeneration of peripheral axons. (ii) Increased intraocular pressure directly injures the optic nerve head mechanically or by disturbing the circulation. (iii) Retinal and optic nerve damage is due to the toxicity of SO. Perhaps a combination of the above-mentioned mechanisms causes optic nerve atrophy. However, surgical damage and the intraocular pressure increase must occur almost equivalently in SO-injected groups and in group G (balanced salt solution injection after gas-mediated vitreous compression). Thus our results suggest that SO uptake by the optic nerve may be one of the major causes of optic nerve atrophy after vitreous surgery with SO. There was no difference in the TEM and EDXA findings between groups M6 and E6. This means that emulsified SO is not different in toxicity and effect on the optic nerve from medical grade SO. Quantitative analysis should be used in further studies of this problem. But we suggest that when the use of $\mathrm{SO}$ is necessary for severe cases, the period of SO tamponade should be as short as possible.

\section{Acknowledgements}

Supported by a Grant-in-Aid for Scientific Research (C2) from the Ministry of Education, Science, Sports and Culture of Japan (grant No. 13671842, 2001-2002).

\section{References}

1 Ando F, Miyake Y, Oshima K, Yamanaka A. Temporary use of intraocular silicone oil in the treatment of complicated retinal detachment. Graefes Arch Clin Exp Ophthalmol 1986; 224: 32-33.

2 Cibis PA, Becker B, Okun E, Canaan S. The use of liquid silicone in retinal detachment surgery. Arch Ophthalmol 1962; 68: 590-599.

3 Ferrone PJ, McCuen BW II, de Juan E Jr, Machemer R. The efficacy of silicone oil for complicated retinal detachments in the pediatric population. Arch Ophthalmol 1994; 112: 773-777.

4 Grey RHB, Leaver PK. Silicone oil in the treatment of massive preretinal traction. I Results in 105 eyes. $\mathrm{Br} \mathrm{J}$ Ophthalmol 1979; 63: 355-360.

5 McCuen BW, Lander MB, Machemer R. The use of silicone oil following failed vitrectomy for retinal detachment with advanced proliferative vitreoretinopathy. Ophthalmology 1985; 92: 1029-1034.

6 Silicone Study Group. Vitrectomy with silicone oil or sulfur hexafluoride gas in eyes with severe proliferative vitreoretinopathy: results of a randomized clinical trial Arch Ophthalmol 1992; 110: 770-779.

7 Silicone Study Group. Vitrectomy with silicone oil or perfluoropropane gas in eyes with severe proliferative vitreoretinopathy: results of a randomized clinical trial. Arch Ophthalmol 1992; 110: 780-792.

8 Eckardt C, Nicolai U, Czank M, Schmidt D. Okulare Gewebe nach intravitrealer Silikonölinjektion. Ophthalmologe 1993; 90: 250-257.

9 Knorr HL, Seltsam A, Holbach L, Naumann GO. Intraocular silicone oil tamponade: a clinico-pathologic study of 36 enucleated eyes. Ophthalmologe 1996; 93: 130138

10 Wenkel H, Nauman GO. Retrolaminar infiltration of optic nerve with intraocular tamponade following silicone oil instillation. Klin Monatsb Augenheilk 1999; 214: 120-122.

11 Eller AW, Friberg TR, Mah F. Migration of silicone oil into the brain: a complication of intraocular silicone oil for retinal tamponade. Am J Ophthalmol 2000; 129: 685688 .

12 Thresher RJ, Ehrenberg M, Machemer R. Gas-mediated vitreous compression: an experimental alternative to mechanized vitrectomy. Graefes Arch Clin Exp Ophthalmol 1984; 221: 192-198.

13 Crisp A, de Juan E Jr, Tiederman J. Effect of silicone oil viscosity on emulsification. Arch Ophthalmol 1986; 105: 546-550.

14 Eckardt C, Nicolai U, Winter M, Knof E. Experimental intraocular tolerance to liquid perfluorooctane and perfluoropolyether. Retina 1991; 11: 375-384.

15 Federman JL, Schubert HD. Complications associated with the use of silicone oil in 150 eyes after retinavitreous surgery. Ophthalmology 1988; 95: 870-876.

16 Gonvers M, Hornung JP, de Courten C. The effect of liquid silicone on the rabbit retina. Arch Ophthalmol 1986; 104: 1057-1057.

17 Laroche L, Pavlakis C, Saraux H, Orcel L. Ocular findings following intravitreal silicone injection. Arch Ophthalmol 1983; 101: 1422-1425.

18 Nakamura K, Refojo MF, Crabtree DF, Pastor J, Leong FL. Ocular toxicity of low molecular weight components of silicone and fluorosilicone oils. Invest Ophthalmol Vis Sci 1991; 32: 3007-3020.

19 Sparrow JR, Chang S. Vitreous substitutes. In: Albert DM, Jakobiec FA (eds). Principles and Practice of Ophthalmology. WB Saunders: Philadelphia, 1994, pp 1142-1159.

20 Doi M, Refojo M. Histopathology of rabbit eyes with silicone-fluorosilicone copolymer oil as six months internal retinal tamponade. Exp Eye Res 1995; 61: 469-478.

21 Ohira A, Chihara E, Soji T. Egress route of emulsified 20 centistokes silicone oil from anterior chamber of rabbit. Curr Eye Res 1993; 13: 489-495.

22 Ohira A, Wilson CA, de Juan E Jr, Murata Y, Shoji T, Oshima K. Experimental retinal tolerance to emulsified silicone oil. Retina 1991; 11: 259-265.

23 Amemiya T, Dake Y. Granuloma after augmentation of the eyelids with liquid silicone: an electron microscopic study. Ophthalmic Reconstr Surg 1994; 10: 51-56. 